\title{
COMPARING PERIODIC ORBITS OF MAPS OF THE INTERVAL
}

\author{
C. BERNHARDT, E. COVEN, M. MISIUREWICZ, AND I. MULVEY
}

\begin{abstract}
Let $\pi$ and $\theta$ be cyclic permutations of finite ordered sets. We say that $\pi$ forces $\theta$ if every continuous map of the interval which has a representative of $\pi$ also has one of $\theta$. We give a geometric version of Jungreis' combinatorial algorithm for deciding in certain cases whether $\pi$ forces $\theta$.
\end{abstract}

\section{INTRODUCTION}

This paper is concerned with the forcing relation between finite cyclic permutations. Let $\pi$ be a cyclic permutation of $\{1, \ldots, k\}$ and let $f: I \rightarrow I$ be a continuous map of a compact interval. A representative of $\pi$ in $f$ is a subset $\left\{x_{1}<\cdots<x_{k}\right\}$ of $I$ such that $f\left(x_{1}\right)=x_{\pi(i)}(i=1, \ldots, k)$. We say that $\pi$ forces $\theta$ if every continuous map of a compact interval which has a representative of $\pi$ also has a representative of $\theta$. The study of the forcing relation is a natural outgrowth of the proof in [BGMY] of Sarkovskii's Theorem.

The formal study of the forcing relation was begun by S. Baldwin [Ba], who showed that forcing is a partial order and gave an algorithm for deciding whether $\pi$ forces $\theta$. Baldwin's algorithm checks, in terms of closed walks through directed graphs with oriented edges (oriented Markov graphs), whether $\theta$ has a representative in the canonical $\pi$-linear map $L_{\pi}$, defined on $[1, k]$ by $L_{\pi}=$ $\pi$ on $\{1, \ldots, k\}$ and $L_{\pi}$ is linear on $[1,2], \ldots,[k-1, k]$. I. Jungreis [J, Theorem 5.1] gave a faster algorithm, in terms of itineraries, for deciding whether $\pi$ forces $\theta$ in certain cases.

In this paper, we present an effective algorithm for deciding whether $\pi$ forces $\theta$ in the cases considered by Jungreis. Our algorithm is in some sense the same as Jungreis'. However, our implementation is in terms of rational numbers instead of itineraries, and our proof is geometric instead of combinatorial, avoiding the "intricate combinatorial theorem" of $[\mathrm{J}, \S 6]$. The main idea of our proof is to compare canonical representatives of $\pi$ and $\theta$ in maps we call horseshoe maps.

Throughout this paper, the lower case Greek letters $\pi, \theta, \eta$ will denote cyclic permutations, and $\pi=\left(a_{1} a_{2} \cdots a_{n}\right)$ means that $\pi\left(a_{1}\right)=a_{2}, \ldots, \pi\left(a_{n-1}\right)=a_{n}$, $\pi\left(a_{n}\right)=a_{1}$. The upper case Latin letters $P, Q, R$ will denote representatives of cyclic permutations, i.e., periodic orbits. The upper case Latin letter $H$ will denote a horseshoe map (defined in the next section).

Received by the editors July 13, 1990.

1980 Mathematics Subject Classification (1985 Revision). Primary 58F20, 58F08, 58H20. 


\section{BACKGROUND}

Let $n \geq 2$. The horseshoe map $H:[0,1] \rightarrow[0,1]$ of type $n^{+}$is defined as follows: for $x \in[i / n,(i+1) / n](i=0, \ldots, n-1)$,

$$
H(x)= \begin{cases}n(x-i / n) & \text { if } i \text { is even, } \\ 1-n(x-i / n) & \text { if } i \text { is odd. }\end{cases}
$$

$H$ is continuous, maps each of the $n$ laps

$$
I_{1}=[0,1 / n], I_{2}=[1 / n, 2 / n], \ldots, I_{n}=[(n-1) / n, 1]
$$

linearly onto $[0,1]$, and is increasing on the first lap. The horseshoe map of type $n^{-}$is defined analogously. It is decreasing on the first lap. In either case, we write $l(H)=n$.

Let $n \geq 0$. We say that $\pi$ has type $n^{+}$(resp. $n^{-}$) if the canonical $\pi$-linear map $L_{\pi}$ has $n$ laps, and is increasing (resp. decreasing) on the first lap. In either case, we write $l(\pi)=n$. Note that the trivial permutation (1) has type $0^{+}$and type $0^{-}$. For any $\pi$ and any $H$, the set of representatives of $\pi$ in $H$ is finite. It is nonempty if and only if $\pi$ and $H$ have the same type or $l(\pi)<l(H)[\mathrm{MN}]$.

An important tool in the early study of forcing was the observation by Baldwin [Ba, Lemma 3.1 and Theorem 3.3] (see also [Be1, Lemmas 1.7 and 1.8]) that if $\theta$ has a representative in the canonical $\pi$-linear map $L_{\pi}$, then $\pi$ forces $\theta$. We shall use a variant of this result involving horseshoe maps.

Suppose $P$ is a periodic orbit of $H$. For $i=0, \ldots, n(n=l(H))$, define $d_{i}=\min \{|p-i / n|: p \in P\}$ and let $\bar{p}_{i}$ be the unique point in $P$ such that $\left|\bar{p}_{i}-i / n\right|=d_{i}$. We say that $P$ fits $H$ if $n=2$ or if $n \geq 3$ and $d_{i}+d_{i+1} \leq 1 / n$ $(i=1, \ldots, n-2)$. In either case, the $P$-truncation $H p$ of $H$, defined by

$$
H_{p}(x)= \begin{cases}H\left(\bar{p}_{i}\right) & \text { if }|x-i / n| \leq d_{i}, \quad(i=0,1, \ldots, n) \\ H(x) & \text { otherwise }\end{cases}
$$

is well defined. The figure below shows the graph of $H p$, where $H$ is the horseshoe map of type $3+$ and

$$
P=\{46 / 365,76 / 365,138 / 365,218 / 365,228 / 365,316 / 365\}
$$

is a representative of $(136425)$ in $H$.

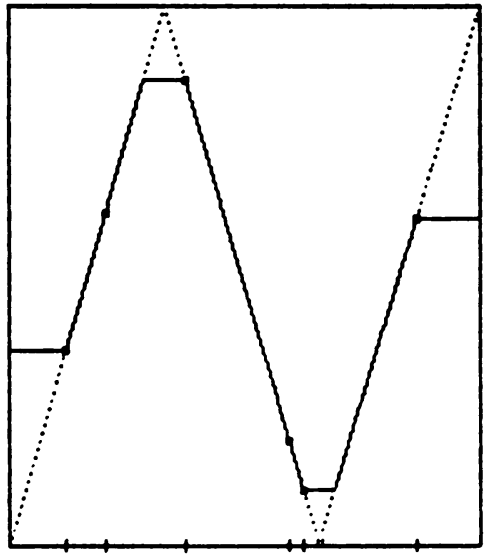


Remark. If $P$ does not fit $H$, then $H_{p}$ may not be well defined. For example, consider the periodic orbit $\{4 / 63,16 / 63,62 / 63\}$ of the horseshoe map of type $4+$.

Lemma 1. Let $P$ be a representative of $\pi$ in $H$ such that $H_{p}$ is well defined. If $\theta$ has a representative in $H_{p}$, then $\pi$ forces $\theta$.

Proof. For $x \in[0,1]$, let $x^{\prime}$ (resp. $x^{\prime \prime}$ ) be the right (resp. left) endpoint of the largest closed interval containing $x$ on which $H_{p}$ is constant. If $P=\left\{x_{1}<\right.$ $\left.\cdots<x_{k}\right\}$, set $x_{0}=0$ and $x_{k+1}=1$. Then every periodic orbit of $H_{p}$ lies in $\bigcup_{i=0}^{k}\left[x_{i}^{\prime}, x_{i+1}^{\prime \prime}\right]$. Therefore by [MN, Theorem 1.21], $\pi$ forces $\theta$.

We say that $\pi$ fits $H$ if the set of representatives of $\pi$ in $H$ is nonempty and every representative of $\pi$ in $H$ fits $H$.

We leave the proof of the following lemma to the reader.

Lemma 2. $\pi$ fits $H$ if and only if one of the following conditions holds:

(1) $\pi$ and $H$ have the same type.

(2) $l(\pi)=l(H)-1$.

(3) $H$ has type $n \pm$ and $\pi$ has type $(n-2) \mp$.

Suppose that $P$ and $Q$ are periodic orbits of $H$ such that $H_{Q}$ is well defined. We say that $P$ is tighter than $Q$ if $H_{Q}|P=H| P$, i.e., $H$ and $H_{Q}$ agree on $P$.

Lemma 3. Suppose that $P$ and $Q$ are periodic orbits of $H$ such that $H_{Q}$ is well defined. Then the following statements are equivalent:

(1) $P$ is tighter than $Q$.

(2) $d_{i}(P) \geq d_{i}(Q)(i=0, \ldots, l(H))$.

If, in addition, $P$ has at least three members, then the following statement is equivalent to the others:

(3) $d_{i}(P) \geq d_{i}(Q)(i=1, \ldots, l(H)-1)$.

Proof. The equivalence of (1) and (2) follows from the definitions. We show that (3) implies (1) if $P$ has at least three members.

Let $n=l(H)$ and let $\bar{H}_{Q}$ be the map defined analogously to $H_{Q}$, except that we do not truncate at 0 or 1 , i.e., we ignore $d_{0}$ and $d_{n}$. Then $P$ is a periodic orbit of $\bar{H}_{Q}$. Since $\bar{H}_{Q}=H_{Q}$ on $\left[1 / n-d_{1},(n-1) / n+d_{n-1}\right]$ and this interval is mapped to itself by $H_{Q}$, it suffices to show that $P \subseteq\left[1 / n-d_{1},(n-1) / n+d_{n-1}\right]$.

The complement of $\left[1 / n-d_{1},(n-1) / n+d_{n-1}\right]$ consists of two intervals, $K_{1}$ and $K_{2} . H$ is linear on these two intervals, either $H\left(K_{1}\right) \cap K_{1}=\varnothing$ or $H\left(K_{1}\right) \cap K_{2}=\varnothing$, and either $H\left(K_{2}\right) \cap K_{1}=\varnothing$ or $H\left(K_{2}\right) \cap K_{2}=\varnothing$. Therefore any periodic orbit of $H$ which lies in $K_{1} \cup K_{2}$ can have only one or two members.

In order to use Lemmas 1 and 3 effectively, we need a method of constructing representatives of a cyclic permutation in a horseshoe map. The tool we shall use is itineraries.

An $H$-itinerary (itinerary for short when $H$ is clear) of a point $x \in[0,1]$ is a sequence $\tau=(\tau(j))_{j=0}^{\infty}$ such that $H^{j}(x) \in I_{\tau(j)}(j=0,1, \ldots)$. The correspondence between points and itineraries is one-to-one, except for those points whose orbit meets the set of turning points of $H$. Such points have two itineraries. Since $H$ maps the turning points to 0 or 1 , this cannot happen for 
periodic points. Thus there is a one-to-one correspondence between periodic points and periodic itineraries. This correspondence preserves (least) periods.

Itineraries are ordered in the following way. Define $\varepsilon_{k}=+1$ (resp. -1$)$ if $H$ is increasing (resp. decreasing) on $I_{k}, \zeta(0)=+1$, and $\zeta(k)=\varepsilon_{\tau(0)} \cdots \varepsilon_{\tau(k-1)}$ $(k \geq 1)$. If $\tau \neq \sigma$ are itineraries, let $k$ be the least integer such that $\tau(k) \neq$ $\sigma(k)$. Then $\tau<\sigma$ if $\zeta(k)=+1$ and $\tau(k)<\sigma(k)$, or if $\zeta(k)=-1$ and $\tau(k)>\sigma(k)$. In the other two cases, $\tau>\sigma$.

Lemma 4 (cf. [MT, Lemma 3.1]). Let $x$ and $y$ be points in [0,1] having unique itineraries $\tau$ and $\sigma$. Then $x<y$ if and only if $\tau<\sigma$.

Theorem 5. Let $l(H)=n$ and let $\tau$ be a sequence with $\tau(i) \in\{1, \ldots, n\} \quad(i=$ $0,1, \ldots)$. Then the unique point in $[0,1]$ whose itinerary is $\tau$ is $\sum_{j=0}^{\infty} \alpha_{j} / n^{j+1}$, where $\alpha_{j}=\tau(j)-1$ if $\zeta(j)=+1, \alpha_{j}=n-\tau(j)$ if $\zeta(j)=-1$.

Proof. Using induction on $m$, it is straightforward to show that for $m \geq 0$, the left endpoint of $I_{\tau(0)} \cap H^{-1}\left(I_{\tau(1)}\right) \cap \cdots \cap H^{-m}\left(I_{\tau(m)}\right)$, which has length $1 / n^{m+1}$, is $\sum_{j=0}^{m} \alpha_{j} / n^{j+1}$.

We say that $\pi$ is a doubling of $\eta$ if for some even $k, \pi$ is defined on $\{1, \ldots, k\}, \eta$ is defined on $\{1, \ldots, k / 2\}$, and $\pi\{2 i-1,2 i\}=\{2 \eta(i)-$ $1,2 \eta(i)\} \quad(i=1, \ldots, k / 2)$. In this case, we write $\eta=\pi / 2$.

Lemma 6 [Be2, Theorem 1.12].

(1) $\pi$ forces $\pi / 2$.

(2) If $\pi$ forces $\theta \neq \pi$, then $\pi / 2$ forces $\theta$.

\section{THE ALGORITHM}

In the propositions below, $\pi$ is defined on $\{1, \ldots, k\}, \pi$ fits $H$, and $n=$ $l(H)$. Label the laps of $L_{\pi}$ from left to right as follows:

(1) $J_{1}, \ldots, J_{n}$ if $H$ and $\pi$ have the same type,

(2) $J_{1}, \ldots, J_{n-1}$ if $H$ has type $n \pm$ and $\pi$ has type $(n-1) \pm$,

(3) $J_{2}, \ldots, J_{n}$ if $H$ has type $n \pm$ and $\pi$ has type $(n-1) \mp$,

(4) $J_{2}, \ldots, J_{n-1}$ if $H$ has type $n \pm$ and $\pi$ has type $(n-2) \mp$.

In cases (2), (3), and (4), we allow $L_{\pi}$ to have "phantom" laps $J_{1}$ and/or $J_{n}$. In this way, $L_{\pi}$ is increasing or decreasing on $J_{m}$ according as $H$ is increasing or decreasing on $I_{m}(m=1, \ldots, n)$.

We define below an itinerary $\tau$ which is the largest $L_{\pi}$-itinerary of 1 , even allowing "phantom" itineraries, where the laps of $L_{\pi}$ are labelled as above.

If $\pi=(1)$, then $H$ has type $2 \pm$. In this case, let $\tau=111 \cdots$ if $H$ has type $2-$, and $\tau=222 \cdots$ if $H$ has type $2+$.

If $\pi \neq(1)$, we define $\tau$ inductively as follows. Let $\tau(0)=1$ if (1) or (2) hold, $\tau(0)=2$ if (3) or (4) hold. Assume that $\tau(0), \ldots, \tau(j-1)$ have been defined. If $L_{\pi}^{j}(1) \in J_{i}$ only, let $\tau(j)=i$. If $L_{\pi}^{j}(1) \in J_{i} \cap J_{i+1}$, let $\tau(j)=i$ if $\zeta(j)=-1, \tau(j)=i+1$ if $\zeta(j)=+1$. (Here $1 \in J_{1} \cap J_{2}$ in cases (3) and (4), and $k \in J_{n-1} \cap J_{n}$ in cases (2) and (4).)

Let $r$ be the unique point in $[0,1]$ whose $H$-itinerary is $\tau$, and let $R(\pi)$ be the orbit of $r$. Since $\tau$ is an $L_{\pi}$-itinerary of $1, r=\min R(\pi)$. In the sequel, $\zeta$ corresponds to the itinerary $\tau$ defined above.

Proposition 7 below is straightforward. 
Proposition 7. If $L_{\pi}$ has a turning point at $L_{\pi}^{j}(1)$ which is a relative maximum (resp. minimum), then $\zeta(j+1)=-1$ (resp. +1$)$.

(Here 1 is a turning point of $L_{\pi}$ in cases (3) and (4), and $k$ is a turning point in cases (2) and (4).)

Proposition 8. $R(\pi)$ is a representative of $\pi$ or $\pi / 2$.

Proof. The result is true for $\pi=(1)$. If $\pi=(12)$, the result is true since $\tau=111 \cdots$ if $H$ has type $2-$ and $\tau=222 \cdots$ if $H$ has type $2+$ and $3+$. So assume that $\pi \neq(1)$ or (12), and hence that $k \geq 3$.

We show that the period of $R(\pi)$ divides $k$. To do this, it suffices to show that $\zeta(k)=+1$, for then $\tau$ and hence $r$ have periods which divide $k$. Since $L_{\pi}^{k}(1)=1$, either $L_{\pi}$ has a turning point at $L_{\pi}^{k-1}(1)$ which is a relative minimum, or $L_{\pi}^{k-1}(1)=k \in J_{n}$ and $L_{\pi}$ is decreasing on $J_{n}$. In the first case, $\zeta(k)=+1$ by Proposition 7 . In the second case, $\varepsilon_{\tau(k-1)}=-1$ and $L_{\pi}$ has a turning point at $L_{\pi}^{k-2}(1)$ which is a relative maximum. Therefore by Proposition $7, \zeta(k-1)=-1$ and so $\zeta(k)=+1$.

Since $k \geq 3, R(\pi)$ cannot lie on a single lap of $H$, and therefore the period of $R(\pi)$ is greater than one. This period is $k / s$, where $1 \leq s<k$. Now $L_{\pi}$ permutes the blocks $\{1, \ldots, s\},\{s+1, \ldots, 2 s\}, \ldots,\{k-s+1, \ldots, k\}$, and is monotone on each block. Therefore $s=1$ or 2 .

The map $\varphi:\{1, \ldots, k\} \rightarrow R(\pi)$, defined by $\varphi(i)=H^{J}(r)$, where $i=$ $L_{\pi}^{j}(1)$, preserves the order relation $\leq$. Thus $R(\pi)$ is a representative of $\pi$ or $\pi / 2$ according as $s=1$ or 2 .

Proposition 9. $R(\pi)$ is a representative of $\pi / 2$ or $\pi$ according as $\pi$ is a doubling or not.

Proof. If $\pi$ is not a doubling, then $\pi / 2$ is not defined, and so by Proposition $8, R(\pi)$ is a representative of $\pi$. Suppose then that $\pi$ is a doubling. Let $L_{\pi}^{j}(1) \in J_{1} \cap J_{i+1}$, where $0 \leq j \leq k / 2$. If $L_{\pi}^{j+k / 2}(1)<L_{\pi}^{j}(1)$, then $\zeta(j)=-1$ and $\zeta(j+k / 2)=+1$, while if $L_{\pi}^{j+k / 2}(1)>L_{\pi}^{j}(1)$, then $\zeta(j)=+1$ and $\zeta(j+$ $k / 2)=-1$. In either case, $\tau(j+k / 2)=\tau(j)$. Therefore $\tau$ and hence $r$ have period $k / 2$. Thus $R(\pi)$ is not a representative of $\pi$, and so by Proposition 8 it must be a representative of $\pi / 2$.

Proposition 10. If $\pi$ is a doubling, then $\zeta(k / 2)=-1$.

Proof. Setting $j=k / 2$ in the proof of Proposition 9, we get $L_{\pi}^{k}(1)<L_{\pi}^{k / 2}(1)$, and hence $\zeta(k / 2)=-1$.

Proposition 11. $R(\pi)$ fits $H$.

Proof. We need only consider the case that $\pi$ is a doubling. We may assume that $n \geq 3$, for if $n=2$ there is nothing to prove.

By Proposition 10, $\zeta(k / 2)=-1$. Then by [MN, Proposition 9.8(1)], the orbit $P$ of the largest fixed point of $H^{k}$ less than $r$ (such exists) is a representative of a cycle $\eta$ which is a doubling of $\pi / 2$ and such that each point of $R(\pi)$ is surrounded by two points of $P$, in the sense that its nearest neighbors in $R(\pi) \cup P$ both are in $P$. Furthermore, if $R(\pi)=\left\{r_{1}<\cdots<r_{k / 2}\right\}$, then $\eta$ is the unique doubling of $\pi / 2$ such that $\eta(2 i)-\eta(2 i-1)$ has the same sign as the slope of $H$ at $r_{i}(i=1, \ldots, k / 2)$. Since $\varepsilon_{\tau(j)}=+1$ or -1 
according as $L_{\pi}^{j+k / 2}(1)-L_{\pi}^{j}(1)$ and $L_{\pi}^{j+k / 2+1}(1)-L_{\pi}^{j+1}(1)$ have the same sign or opposite signs $(j=0, \ldots, k / 2-1), \pi$ has this last property, and so $P$ is a representative of $\pi$.

If $P=\left\{p_{1}<\cdots<p_{k}\right\}$, call the points $p_{2 m-1}$ and $p_{2 m} \quad(m=1, \ldots, k / 2)$ partners. If $d_{i}(P)$ is attained at $\bar{p}_{i} \in P$, let $\tilde{p}_{i}$ be the partner of $\bar{p}_{i}$, and let $\tilde{d}_{i}=\left|\tilde{p}_{i}-i / n\right|$. Since $P$ fits $H, d_{i}+d_{i+1} \leq 1 / n \quad(i=1, \ldots, n-2)$. Using the fact that $H$ maps partners to partners, it is straightforward to show that $\tilde{d}_{i}+\tilde{d}_{i+1} \leq 1 / n(i=1, \ldots, n-2)$ as well. Since the point of $R(\pi)$ at which $d_{i}(R(\pi))$ is attained lies between $\bar{p}_{i}$ and $\tilde{p}_{i}(i=0, \ldots, n)$, we have $d_{i}(R(\pi))+d_{i+1}(R(\pi)) \leq 1 / n \quad(i=1, \ldots, n-2)$.

Remark. If $\pi$ is a doubling which fits $H$, then although $R(\pi)$ fits $H$, some other representative of $\pi / 2$ may not fit $H$. For example, $\pi=(136245)$, of type $3+$, fits the horseshoe map $H$ of type $4+$, but $\pi / 2=(123)$ has a representative which does not fit $H$. See the remark before Lemma 1.

Proposition 12. $R(\pi)$ is tighter than every representative of $\pi$ in $H$.

Proof. The result is trivial if $\pi=(1)$, so we may assume that $k \geq 2$. By looking at the points $\left(i, L_{\pi}(i)\right)(i=1, \ldots, k)$ in the graph of $L_{\pi}$ and the points $(p, H(p))(p \in P)$ in the graph of $H$, it is easy to see that for every representative $P$ of $\pi$ in $H$, and for $P=R(\pi)$ as well if $\pi$ is a doubling, $d_{i}(P)$ is attained at $H^{j}(p)$, where $p=\min P$ and $j$ is the unique integer, $0 \leq j \leq k-1$, such that $L_{\pi}^{j}(1) \in J_{i} \cap J_{i+1} \quad(i=1, \ldots, n-1)$. Therefore to show that $d_{i}(R(\pi)) \geq d_{i}(\dot{P})$, it suffices to show that $H^{j+1}(p) \geq H^{j+1}(r)$ (resp. $\left.H^{j+1}(p) \leq H^{j+1}(r)\right)$ if $L_{\pi}$ has a relative maximum (resp. minimum) at $L_{\pi}^{j}(1)$.

The itinerary $\sigma$ of $p$ is also an $L_{\pi}$-itinerary of 1 . From the construction of $\tau$ and the definition of ordering of itineraries it follows that at each step of the construction of $\tau$ we make the choice that makes $\tau$ maximal. Hence $\sigma \leq \tau$ and so $p \leq r$. By Proposition 7, if $L_{\pi}$ has a relative maximum at $L_{\pi}^{j}(1)$, then $H^{j+1}(p) \geq H^{j+1}(r)$, and if $L_{\pi}$ has a relative minimum at $L_{\pi}^{j}(1)$, then $H^{j+1}(p) \leq H^{j+1}(r)$. Therefore $d_{i}(P) \leq d_{i}(R(\pi)) \quad(i=1, \ldots, n-1)$, and $R(\pi)$ is tighter than $P$ by Lemma 3.

Remark. It can be shown that if $\pi$ is a doubling and $l(\pi) \geq 3$, then $\pi$ has no tightest representative in the horseshoe map of the same type as $\pi$. The simplest example is $\pi=(136245)$.

Theorem 13 (cf. [J, Theorem 5.1]). Let $\pi$ and $\theta$ fit $H$. Then $\pi$ forces $\theta$ if and only if $R(\theta)$ is tighter than $R(\pi)$.

Proof. By Proposition $11, R(\pi)$ fits $H$, and so $H_{R(\pi)}$ is well defined.

Assume that $\pi$ forces $\theta$. Suppose first that $\pi$ is not a doubling. Then $R(\pi)$ is a representative of $\pi$ in $H$, and therefore $\theta$ has a representative $Q$ in $H_{R(\pi)}$. Then $Q$ is a representative of $\theta$ in $H$ and $Q$ is tighter than $R(\pi)$. But $R(\theta)$ is tighter than $Q$ by Proposition 12, so $R(\theta)$ is tighter than $R(\pi)$. Suppose now that $\pi$ is a doubling. We may assume that $\theta \neq \pi$. By Lemma 6 , $\pi / 2$ forces $\theta$. By Proposition $9, R(\pi)$ is a representative of $\pi / 2$ in $H$, and the argument above shows that $R(\theta)$ is tighter than $R(\pi)$.

Conversely, assume that $R(\theta)$ is tighter than $R(\pi)$. If $\theta$ is not a doubling, then $R(\pi)$ and $R(\theta)$ are representatives of $\pi$ ( $\pi / 2$ if $\pi$ is a doubling) and $\theta$ 
in $H_{R(\pi)}$, and it follows from Lemma 1 that $\pi$ ( $\pi / 2$ if $\pi$ is a doubling) forces $\theta$. In the parenthetical case too, $\pi$ forces $\theta$, because $\pi$ forces $\pi / 2$. Suppose then that $\theta$ is a doubling. By Proposition $10, \zeta^{\prime}\left(k^{\prime} / 2\right)=-1$ (here the primes refer to $\theta$ ), so as in the proof of Proposition 11 , there is a representative of $\theta$ in $H_{R(\pi)}$. As above, $\pi$ forces $\theta$.

If $\tau$ is periodic, then the terms of the series in Theorem 5 can be grouped to give a geometric series. Thus $R(\pi)$ consists of rational numbers which are computable in finitely many steps, as does the set of numbers $\left\{d_{i}(R(\pi))\right\}$. Therefore

If $H$ is the horseshoe map of the same type as $\pi$, then Theorem 5 and 13 together give an effective algorithm for deciding whether $\pi$ forces $\theta$ in each of the following cases:

(1) $\pi$ and $\theta$ have the same type,

(2) $l(\theta)=l(\pi)-1$,

(3) $\pi$ has type $n \pm$ and $\theta$ has type $(n-2) \mp$.

Remark. If $\pi$ is a doubling, we can sometimes decide whether $\pi$ forces $\theta$ even if $\theta$ does not fit the horseshoe map of the same type as $\pi$. Formally, define $\pi_{0}=\pi$, and if $\pi_{i}$ is a doubling, define $\pi_{i+1}=\pi_{i} / 2$ : if not, $\pi_{i}$ is not defined. Let $m$ be the smallest integer such that $\pi_{m}$ is not a doubling. To decide whether $\pi$ forces $\theta$, first check whether $\theta=\pi_{i}$ for some $i \leq m$. If so, then $\pi$ forces $\theta$ by Lemma 6 ; if not, then again by Lemma $6, \pi$ forces $\theta$ if and only if $\pi_{m}$ forces $\theta$. The algorithm may apply to $\pi_{m}$ and $\theta$. For example, (171145102812369), of type $8+$, forces (13425) but not (1234), both of type $2+$.

\section{REFERENCES}

[Ba] S. Baldwin, Generalizations of a theorem of Sarkovskii on orbits of continuous real-valued functions, Discrete Math. 67 (1987), 111-127.

[Be1] C. Bernhardt, Simple permutations with order a power of two, Ergodic Theory Dynamical Systems 4 (1984), 179-186.

[Be2] _ The ordering on permutations induced by continuous maps of the real line, Ergodic Theory Dynamical Systems 7 (1987), 155-160.

[BGMY] L. Block, J. Guckenheimer, M. Misiurewicz, and L.-S. Young, Periodic points and topological entropy of one-dimensional maps, Lecture Notes in Math., vol. 819, Springer, 1980, pp. 18-34.

[J] I. Jungreis, Some results on the Sarkovskii partial ordering of permutations, Trans. Amer. Math. Soc. (to appear).

[MT] J. Milnor and W. Thurston, On iterated maps of the interval, Lecture Notes in Math., vol. 1342, Springer, 1988 , pp. $465-563$.

[MN] M. Misiurewicz and Z. Nitecki, Combinatorial patterns for maps of the interval, Universität Göttingen, preprint, 1989.

(C. Bernhardt and I. Mulvey) Department of Mathematics and Computer Science, FairFIELD UNIVERSITY, FAirfield, CONNECTICUT 06430

E-mail address: cbernhard@fairl.bitnet

E-mail address: itmulvey@fair1.bitnet

(E. Coven) Department of Mathematics, Wesleyan University, Middletown, ConnectiCUT 06457

E-mail address: coven@jordan.math.wesleyan.edu

(M. Misiurewicz) Instytut Matematyki, Uniwersytet Warszawski, 00-901 Warsaw, POLAND 